\title{
CrimRxiv
}

\section{Gated Adaptation during the Cape Town Drought: Mentalities, Transitions and Pathways to Partial Nodes of Water Security}

Nicholas P. Simpson, Clifford Shearing, Benoit Dupont

Published on: Jun 21, 2021

License: Creative Commons Attribution 4.0 International License (CC-BY 4.0). 
